\title{
Good Prescribing Interventions, made an Impact in Reduced Coartem Usage - a Retrospective Study at the Copperbelt University Health Facility
}

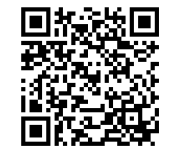

\author{
Kaselekela Ponshano* \\ Copperbelt University, Zambia
}

Submission: May 16, 2018; Published: May 29, 2018

*Corresponding author: Ponshano Kaselekela, Copperbelt University, Zambia; Tel: +260-964-921932; Email: ponshkase@cbu.ac.zm

\begin{abstract}
In Zambia, the first line drug in the treatment of confirmed positive cases of Malaria is Artemether plus lumefantrine (Coartem). The retail cost of these drug per course ranges from $\$ 3.50$ to $\$ 4.50$ in Zambia which is very expensive to the organisation as well as to a common man. However, irrational prescribing of coartem was identified as the major problem at the Copperbelt University health facility. A study to establish the usage of coartem was conducted for a period of six months from July to December, 2017. The first and the last three months was to establish how this valuable drug was being prescribed before and after the prescribing interventions were introduced respectively. A total of 1,143 courses of coartem were prescribed during the study period. Initially 552,168 and 264 courses of coartem were used in the first three months of the study respectively. After the prescribing interventions were implemented, 54, 50 and 55 courses of coartem were used in the last three months.

A register book was opened at the pharmacy were all clients who were prescribed with coartem were recorded. The information included in the register book included the date at which the medicines were dispensed, name of patient, parasitological test results (microscopy or rapid diagnostic test), dose of coartem supplied and the name of prescriber. Coartem usage was observed to have reduced by $90.04 \%$ at the end of the study. This was after the prescribing interventions were introduced hence reduced costs in terms coartem procurement.
\end{abstract}

Keywords: Good prescribing; Monitoring; Rational drug use; Coartem; Dispensing

Abbreviations: ABC: Artemisin Based Combination; CBU: Copperbelt University; (RDTs): Rapid Diagnostic Tests for Malaria; MOH: Ministry of Health; CCRB: Coartem Consumption Register Book; (ITNs): Insecticide Treated Mosquito Nets; DTC: Drug and Therapeutics Committee

\section{Introduction}

According to the WHO malaria report, 2017, it was reported in 2016 that, 91 countries had a total of 216 million cases of malaria of which malaria deaths globally reached 445,000 [1]. Malaria is the commonest presentation illness in Sub-Sahara Africa, and over diagnosis of malaria in patients presenting with fever in Zambia is a major public health problem [1-3]. Malaria remains an important cause of illness and death in adults and children. It requires an integrated approach, including prevention and prompt treatment with effective antimalarial agents [1-3]. The recommended first line treatment of malaria in Zambia is artemisin based combination ( $\mathrm{ABC}$ ) therapy that is Artemether $20 \mathrm{mg}+$ Lumefantrine $120 \mathrm{mg}$ (coartem) tablets [2]. Over the past years, the introduction of the relatively expensive artemether plus lumefantrine (Coartem) drug and the subsequent appearance of malaria resistance in our country have driven the need to rationalize the overuse of antimalarial drugs at the Copperbelt University (CBU) health facility [3].
Furthermore, the recent decline in malaria transmission in many areas of Zambia has led to increasing awareness that is often associated with neglect of non-malarial causes of fever [46]. The availability of rapid diagnostic tests for malaria (RDTs) as well as parasitological test (microscopy) has provided a potentially important instrument to address these problems. All suspected cases of fever should have malaria tests to confirm the diagnosis by using both microscopy and RDTs and supported by a quality assurance programme. In 2015 the 'WHO Guidelines for Malaria Diagnosis and Treatment' recommended parasitological confirmation in all patients with suspected malaria before treatment and restricting antimalarial treatment to parasite-positive patients $[7,8]$. This was one of the managerial interventions that were implemented at CBU health facility. The idea was to limit the over use of valuable drug to patients who may not need them but due to the presenting fever, physicians tend to prescribe even when the microscopic examination 
for malaria parasite have not be done. The cost of one course of coartem tablets or suspension for adult and children in Zambia ranges from $\$ 3.50$ to $\$ 4.50$ which is relatively very expensive. Over prescribing of this drug can be very costly to the organization financing the procurement of health commodity as well as to the patient.

Providing new tools and instruments does not however necessarily change the prescriber practice. However, increased use of parasitological tests of malaria to guide treatment options often fails to achieve its objective due to some patient's desire and social support for denying ant malarial drugs, leading to a persistent preference among prescribers for a diagnosis of malaria, even in an event of a negative test result. In 1997 increased availability of blood slide microscopy in Zambia had little effect due to the tendency to prescribe ant malarial treatment on clinical diagnosis ('presumptive treatment') or to slide-negative patients. A number of more recent studies have shown that prescriber have more prevalence on clinical diagnosis than laboratory investigations of malaria. This reduces both the clinical effectiveness and cost-effectiveness of RDTs and leads to persistent over diagnosis of malaria which results to wastage of valuable medicine. Getting misdiagnosis of febrile illness as malaria close to zero should lead to improved case management as well as reduced coartem unnecessary usage. The reasons for over prescription of coartem even when test results are showing negative are complex, and depend on prescriber perceptions including of patient expectations [4-6]. Therefore interventions aimed at patient perceptions as well as prescribers need to prescriber coartem in negative clients should be addressed and reinforced especially targeting prescribers.

\section{Objectives}

The objectives of the study include: i. To establish the overall usage of artemether plus lumefantrine (Coartem) drug at the CBU health facility.

ii. To know and establish the drug usage after good prescribing interventions were introduced

iii. To promote rational drug use.

iv. To sensitize prescribers on the importance of prescribing coartem using laboratory investigations.

v. To promote the use of parasitological tests and Rapid diagnostic tests in the treatment of malaria

\section{Methodology}

CBU health facility receives antimalarial drugs in particular coartem from Kitwe district health office through the Ministry of Health (MOH) of the Government of the Republic of Zambia. In June, 2017, there was a concern from the district health office that out of 17 health facilities in Kitwe, CBU health was the first in terms of coartem consumption even when the catchment population of the facility is low. From July to December, 2017 we conducted a study at CBU health facility situated with riverside area in Kitwe, Zambia to establish coartem consumption and how it was being prescribed. At the commencement of the study, prescribers were prescribing freely for clinical and confirmed malaria. It was up to their jurisdiction to prescribe the antimalarial drugs even if the malaria slide was negative. To have consumption data on the usage of coartem at the CBU health facility, the previous three months dispensed prescriptions were analyzed. The data below shows the consumption pattern before the prescribing interventions guidelines on the use of coartem were introduced (Table 1). From September to Deccember, 2017, the health facility management implemented some interventions and guidelines on how coartem must be prescribed as well as being dispensed to the clients. These interventions included.

Table 1: Coartem consumption vs lab. Results.

\begin{tabular}{|c|c|c|c|c|c|}
\hline \multirow{2}{*}{ Month } & \multirow{2}{*}{$\begin{array}{c}\text { Type of } \\
\text { Artemether+lumefantrine } \\
\text { (Coartem) }\end{array}$} & \multirow{2}{*}{ No. of Courses } & \multicolumn{2}{|c|}{ Laboratory Results } & \multirow{2}{*}{ Total } \\
\hline & & & Negative & Positive & \\
\hline \multirow{4}{*}{ July, 2017} & $20 m g+120 m g$ & 24 & & & \\
\hline & $40 \mathrm{mg}+240 \mathrm{mg}$ & - & 546 & 6 & 552 \\
\hline & $60 \mathrm{mg}+360 \mathrm{mg}$ & 264 & & & \\
\hline & $80 \mathrm{mg}+480 \mathrm{mg}$ & 264 & & & \\
\hline \multirow{4}{*}{ August, 2017} & $2 \mathrm{omg}+120 \mathrm{mg}$ & 144 & & & \\
\hline & $40 \mathrm{mg}+240 \mathrm{mg}$ & - & 165 & 3 & 168 \\
\hline & $60 \mathrm{mg}+360 \mathrm{mg}$ & - & & & \\
\hline & $80 \mathrm{mg}+480 \mathrm{mg}$ & 24 & & & \\
\hline \multirow{5}{*}{ September, 2017} & $2 \mathrm{omg}+120 \mathrm{mg}$ & 24 & & & \\
\hline & $40 \mathrm{mg}+240 \mathrm{mg}$ & 48 & 258 & 6 & 264 \\
\hline & $60 \mathrm{mg}+360 \mathrm{mg}$ & 24 & & & \\
\hline & $80 \mathrm{mg}+480 \mathrm{mg}$ & 168 & & & \\
\hline & & & & & 984 \\
\hline
\end{tabular}


A. Introduction of Coartem Consumption Register Book (CCRB), which was maintained at the health facility. The data entered in the CCRB included:
a) Date when the drug was prescribed,
b) Name of the patient who had been prescribed with coartem,

c) The clinic card number and age of the patient,

d) Laboratory Malaria Results (RDTs or Parasitological test),
e) The dose of Coartem prescribed and
f) Name of the medical professional who prescribed the medicine.

B. All prescriptions with coartem on it should be accompanied by the laboratory malaria results before it can be dispensed.

C. To hold clinical meetings every month to monitor and evaluate the coartem usage and to make other possible interventions

D. A monthly report on the consumption of the drug is submitted to the district health for further analysis.

\section{Operational definitions}

Prescribing: An art (of a medical practitioner) advise and authorize the use of a medicine for someone, especially in writing. Prescribing can be good or bad depending on the prescriber if he or she is able to adhere to standard treatment guidelines or not [9-11].

Table 2: Coartem consumption vs lab. results.

\begin{tabular}{|c|c|c|c|c|c|}
\hline \multirow{2}{*}{ Month } & \multirow{2}{*}{$\begin{array}{c}\text { Type of } \\
\text { Artemether+lumefantrine } \\
\text { (Coartem) }\end{array}$} & \multirow{2}{*}{ No. of Courses } & \multicolumn{2}{|c|}{ Laboratory Results } & \multirow{2}{*}{ Tota } \\
\hline & & & Negative & Positive & \\
\hline \multirow{4}{*}{ October, 2017} & $2 \mathrm{omg}+120 \mathrm{mg}$ & 12 & & & \\
\hline & $40 \mathrm{mg}+240 \mathrm{mg}$ & 5 & 38 & 16 & 54 \\
\hline & $60 \mathrm{mg}+360 \mathrm{mg}$ & 1 & & & \\
\hline & $80 \mathrm{mg}+480 \mathrm{mg}$ & 35 & & & \\
\hline \multirow{4}{*}{ November, 2017} & $2 \mathrm{omg}+120 \mathrm{mg}$ & 9 & & & \\
\hline & $40 \mathrm{mg}+240 \mathrm{mg}$ & 7 & 30 & 20 & 50 \\
\hline & $60 \mathrm{mg}+360 \mathrm{mg}$ & 5 & & & \\
\hline & $80 \mathrm{mg}+480 \mathrm{mg}$ & 29 & & & \\
\hline \multirow{5}{*}{ December, 2017} & $2 \mathrm{omg}+120 \mathrm{mg}$ & 3 & & & \\
\hline & $40 \mathrm{mg}+240 \mathrm{mg}$ & 2 & 50 & 5 & 55 \\
\hline & $60 \mathrm{mg}+360 \mathrm{mg}$ & 1 & & & \\
\hline & $80 \mathrm{mg}+480 \mathrm{mg}$ & 48 & & & \\
\hline & & & & & 159 \\
\hline
\end{tabular}

Coartem: is known as an artemisinin-based combination therapy (ACTs) which contains 2 active substances, artemether and lumefantrine that work together to eliminate malaria parasite. It's a prescription drug used to treat acute uncomplicated malaria infections due to Plasmodium falciparum in adults and children [9].

Dispensing: It involves interpretation, evaluation and implantation of a prescription including the preparation and supply the drug with clear instructions to the patient [10].

Prescription: is an order to prepare or dispense a specific treatment usually a medicine to a named patient $[12,13]$.

Rational drug use: is the appropriate way of prescribing medication to the patient according to their clinical needs, in doses that meets them for an adequate period of time at the lowest cost to them and the community [11].

Interventions: These are actions taken to rectify a problem or system disorder.

\section{Ethical approval}

The study was solemnly for information as well as educational. There was no ethical approval granted by any ethical committee in Zambia.

\section{Results and Findings}

It was found that the number of patients being prescribed with Coartem was drastically reduced in the last three months after the prescribing interventions was instituted. This represented $84.23 \%$ reduction as compared to when there were no interventions on prescribing coartem. Table 2 below shows the outcome or scenario. 
The idea of bringing about those interventions was to protect the valuable drug from being given to patients who really do not need it. However, the Zambian governments under the ministry of Health previously were allowing prescribers on the benefit of doubt to prescribe an antimalarial if the patient body temperature was above 37 degrees Celsius for over 24 hours or more even when the parasitological test or RDT comes out to be negative. In terms cost implication, it was observed that there was a decrease in the amount of money being spent in procuring coartem for the health facility. The institution drug budget on procuring this drug will be reduced. For instance, in July, 2017 the health centre used 552 courses of coartem which translates to $\$ 2,484$ while in December, 2017 the amount of antimalarial used was 55 and the cost per month was $\$ 247.50$. Over $90 \%$ of the drug budget can be reduced if the standard treatment guidelines for coartem can be adhered to. The number of patients who were prescribed with coartem but had negative malaria parasite where reduced as compared to those with positive malaria parasite. This showed that after the policy interventions on prescribing the antimalarial, prescribers started prescribing the drug if the laboratory results (parasitological test or RDTs) come out positive.

\section{Discussion}

It was confirmed that prescribers can be influenced by the decisions made by their superiors as well as from the viable and committed drug and therapeutic committees in respective health facilities to monitor and evaluate the use of health commodities [14].

This study can be elaborated and discussed in four (4) areas.

\section{Good prescribing interventions}

Inappropriate prescribing is a result of irrational drug use that occurs when medicines are not prescribed in accordance with guidelines based on scientific evidence to ensure safe, effective, and economic use. The main reasons for such practices on the part of prescribers and consumers need to be understood and addressed in any good intervention. The initial step in developing a strategy to address irrational prescribing practices must be to consider who the prescribers are. In most developed countries, prescribers are doctors or clinical officers who are highly trained in patient management. In many places, however, prescribers may include nurses, paramedics, and drug sellers. The latter, in particular, may have received little or no training in the use of medicines. A comprehensive policy should attempt to influence prescribing behavior at all levels of health delivery system, focusing on the main problems and concentrating on the prescribers involved. However, all the issues on the prescribing pattern can be avoided if the following were put in place.

A. Implementing procedures and guidelines for developing, using, and revising standard treatment guidelines (STGs). Treatment Guidelines have potential benefits that can ensure good prescribing behavior [9]. a. Benefits of Prescribing using Standard Treatment Guidelines

a) For Health Managers

I. Identification of cost -effective treatments for common health problems

II. Basis to assess and compare quality of care

III. Basis for integrating special programs

b) For Supply Management Staff

i. Identification of mostly used drugs for the common ailments

ii. Facilitation of prepackaging of mostly prescribed drugs

iii. Forecasting of drugs required to be consumed at a particular period

c) For health care workers

I. Opportunity for providers to concentrate on correct diagnosis

II. Quality of care standard

III. Come up with most effective, economical treatment for specific setting

IV. Basis for monitoring and supervision

d) For patients

i. Improved adherence to treatment through consistency among prescribers

ii. Most cost effective treatments are provided

iii. Improvement in availability of drugs

iv. Better treatment given to the patients [10-15].

B. Continuing professional development in medical education as a licensure requirement and targeted educational programs by professional societies, universities, and the government.

C. Monitoring, supervision, and using group processes to promote rational medicine use.

D. Monitoring and promoting rational use of medicines.

E. Implementing procedures for developing and revising an essential medicines list (or health facility formulary) based on treatments of choice or disease prevalence $[15,16]$.

F. Avoiding prescribing on financial incentives or gain as this results on over prescribing.

G. Appropriate control of the activities of all those involved in the use of medicines is critical to ensure rational medicine use. 
The framework of good prescribing interventions can be illustrated based on the chart below.

\section{Frame work for formation interventions (Source: Quick et al. 1991)}

Define problems with medicine use pattern

a. Prescribing

\section{Identify motivating factors, underlying causes}
i. Supply logistic
ii. Economic, social
iii. Cultural

\section{List possible interventions}
I. Managerial
II. Educational

\section{Choose possible interventions to implement}
III. Culture acceptance
IV. Cost

\section{Conduct Controlled study of interventions}

From frame work number five, the interventions can either be implemented, revised/Changed or drop ineffective interventions $[11,15]$. For policies to be effective, they must be enforced. Without sufficient competent health personnel, none of the core components of a national program to promote rational use of medicines can produce good results. In managerial interventions, prescribers are guided in the decision-making process, through limiting lists for routine procurements, drug use review and feedback. To reduce the spread of drug resistance to malaria falciparum, and limit the over use of antimalarial drugs particularly coartem should be administered only to patients who truly have confirmed positive cases of malaria. All patients on antimalarial treatment course should be counseled to complete the course to minimize drug resistance.

\section{Effects of inappropriate prescribing}

The overall assessment of Coartem usage showed that the cost implications of irrational prescribing were enormous. However, some gaps existed and attempts were made to resolve those. 552 courses of coartem translated to $\$ 2,484$ as compared to 55 courses of coartem which can cost the institution $\$ 248$ per month. Two thousand thirty six US dollars can be saved monthly and this amount of money can be used to acquire other healthcare needs. In general, inappropriate prescribing of medicines can lead to:

i. Wastage of valuable medicines to patients, who do not really need them,

ii. Adverse drug reactions especially in elderly

iii. Increased healthcare costs,

iv. Drug resistance

v. Difficult in forecasting the drug need due to high demand of the drug,

vi. Patient loses confidence in the health care system (Table 3) (Figure 1).

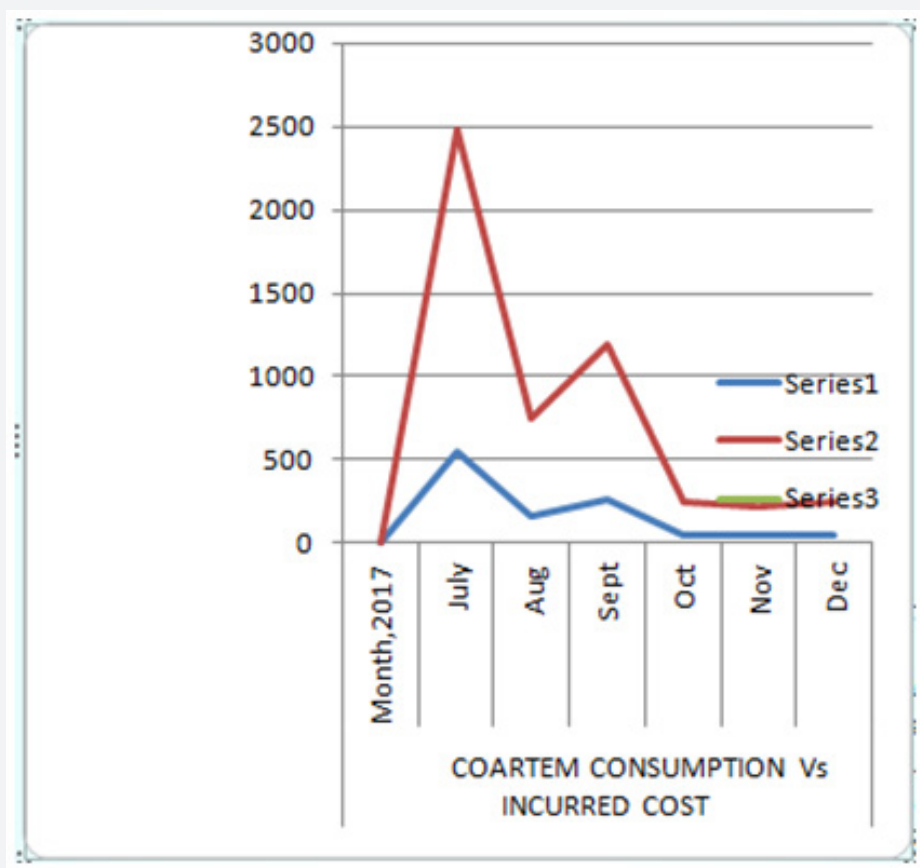

Figure 1: Coartem consumption Vs incurred cost. 
Table 3: Coartem consumption vs incurred cost.

\begin{tabular}{|c|c|c|c|c|c|c|}
\hline Month, 2017 & July & Aug & Sept & Oct & Nov & Dec \\
\hline $\begin{array}{c}\text { No. Coartem } \\
\text { Courses }\end{array}$ & 552 & 168 & 264 & 54 & 50 & 55 \\
\hline Total Cost $(\$)$ & 2,484 & 756 & 1,188 & 243 & 225 & 248 \\
\hline
\end{tabular}

\section{Malaria prevention strategies}

As a country, how have we fared in delivering services that prevent, diagnose and prompt treatment of malaria? At CBU campus, health authorities have made important headway in addressing the challenges of malaria prevention.. However, the pace of progress must be recommended and accelerated if we are to reach our global malaria targets for 2020 and beyond. The CBU management through the office of public health has made a lot of interventions in addressing some of the preventive measures in preventing malaria infection in the institution another surrounding area. In 2016, about (54\%) of people at risk of malaria in sub-Saharan Africa and at CBU health facility the following malaria preventive measures have been implemented:

I. Sleeping under an insecticide-treated mosquito net which is one of the primary prevention methods. Encouraging people or sensitizing the community on the importance sleeping under insecticide treated mosquito nets (ITNs) is the most effective way of preventing the malaria infections.

II. Spraying inside walls of homes with insecticides (indoor residual spraying, IRS) is another important prevention measure. The report documents a precipitous drop in IRS coverage in the WHO African Region since 2010, as well as declines in all other WHO regions over this same period.

III. Prompt diagnosis and treatment is the most effective means of preventing a mild case of malaria from developing into severe disease and death. In the WHO African Region, most people who seek treatment for malaria in the public health system receive an accurate diagnosis and effective treatment.

However, access to the public health system remains far too low. National-level surveys in the WHO African Region show that only about one third (34\%) of children with a fever are taken to a medical provider in this sector.

\section{Effective drug \& therapeutic committees}

Drug and Therapeutics committee (DTC) is also another avenue that can be used to correct medicines prescribing anomalies in any health established institutions. It has a role of optimizing rational use of medicines by ensuring that the best clinical uses of pharmaceuticals are employed. Some of the benefits of this committee in a health setting include: a) Management and effective control of health commodities expenditures

b) It makes medicine procurement and inventory management more effective,

c) It improves quality of patient care and health outcomes by ensuring that all essential medicines are always in stock,

d) It makes that medicine use problems are corrected appropriately

e) Improved medicine use including antimalarial and other drugs

f) It selects safe, high quality and cost effective medicines for the health institution.

\section{Conclusion}

Medicines are an essential component of health care delivery. When the medicines are being used rationally, they produce desired effects of improving the wellbeing of the patients' disease conditions. Irrational prescribing of medicines can lead to prolongation of illness as well as exacerbation of adverse drug reactions and unnecessary expenditure in medicine procurements. Various strategies can be helpful in minimizing inappropriate prescribing of coartem in health facilities worldwide. These strategies can be employed as one or undertaken simultaneously for the benefit of the health institution.

Currently, many countries in sub-Saharan Africa have adopted ACTs as the first-line treatment of malaria. However, irrational use of coartem is a major problem globally. Therefore, global coordinated action is essential to protect the unnecessary use of these drugs by implementing several intervention measures at each point of health care delivery. Physician must always be mindful in the way they prescribe coartem to their clients by ensuring that only positive parasitological tests will attract coartem supply and patient follow -up should be enhanced in order to detect patients who may require further investigations. If we are considerate as a country in the way we use ACTs, there is a real promise of achieving a sustainable malaria treatment strategy by the year 2030 .

\section{Recommendation}

In order to improve the irrational prescribing at CBU health facility as well as for the country in general, the following 
intervention measures should be adopted and strengthened in future:

I. Continued sensitization of prescribers from all healthcare workers on the standard treatment guidelines for malaria,

II. DTC should be implemented to evaluate and guide the best modalities of medicines use,

III. Promote the public awareness on when coartem can be administered them,

IV. All suspected cases of malaria should be treated in accordance to the standard treatment guidelines for the country,

V. Pharmacists' should always ensure that before dispensing coartem, laboratory results should be availed to them as a prove before the medicine can be supplied [17].

\section{References}

1. WHO (2007): World Malaria report. WHO, Geneva, Switzerland.

2. Amexo M, Tolhurst R, Barnish G, Bates I (2004) Malaria misdiagnosis: effects on the poor and vulnerable. Lancet 364(9448): 1896-1898.

3. WHO (2015) Guidelines further treatment of Malaria. ( $3^{\text {rd }}$ edn), Geneva, Switzerland, pp. 1-318.

4. Zambia Central Board of Health, MOH (2004) Standard treatment guidelines, Essential medicines list and Laboratory Supplies List. $\left(1^{\text {st }}\right.$ edn), Pretoria, South Africa, pp. 1-508.

5. Cundill, Mbakilwa H, IR Chandler C, Mtove G, Mtei F, et al. (2005) BMC medicine; prescriber and patient-oriented behavior interventions to improve use malaria RDTs in Tanzania: facility based cluster randomized trial.
6. Chandler CI, Mangham L, Njei AN, Achonduh O, Mbacham WF, et al. (2012) As a clinician, you are not managing lab results, you are managing the patient': how the enactment of malaria at health facilities in Cameroon compares with new WHO guidelines for the use of malaria tests. Med 74(10): 1528-1535.

7. Ministry of Health \& Family Welfare (2013) Diagnosis \& Treatment of Malaria in India.

8. Choonara I (2013) Rational Prescribing is Important in All Settings.

9. (2018) Norvatis Pharmaceutical Corporation, Manual on Coartem prescribing \& patient information.

10. College of Dental Hygienists of Ontario (2015) GUIDELINE on recommending, prescribing, dispensing, selling and use of drugs in practice.

11. Management Sciences for Health (1985) Rational drug use: prescribing, dispensing, counseling and adherence in ART programme.

12. Anil Kumar (2015) Rational prescribing \& prescription writing: Role of clinical pharmacologist to improve the patient quality of care. Indian Journal of Pharmacy and Pharmacology 2(2): 134-138.

13. Jaine S, Prerna U, Jaswant G, Kumar A, Pushpawati J, et al. (2015) a systematic review of prescription pattern monitoring studies and their effectiveness in promoting rational use of medicines perspectives, clinical research 5(2): 86-90.

14. WHO, MSH, USAID (2007) Drug \& Therapeutics Committee Training Course: Session 1. Drug and Therapeutics Committee-Overview, participants Guide. Geneva, Switzerland, p. 28.

15. WHO (1997) Management Sciences for Health, Managing Drug supply. $\left(2^{\text {nd }}\right.$ edn), revised and expanded, Kumarian Press, Sterling, USA.

16. de Vries TPGM, Henning RH, Fresle DA (1994) Guide to Good prescribing: A practical Manual. Geneva, Switzerland, pp. 1-142.

17. MSH, WHO (1997) Managing Drug Supply. ( $\left.2^{\text {nd }} e d n\right)$, Kumarian Press, Sterling, USA.

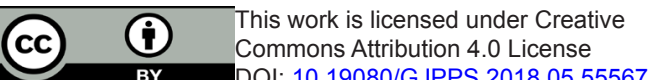

